Communications in Physics, Vol. 24, No.3S2 (2014), pp. 52-62

DOI:10.15625/0868-3166/24/3S2/5060

\title{
MEASUREMENT OF THE UPPER TROPOSPHERIC DENSITY AND TEMPERATURE PROFILES IN HANOI USING A RAMAN LIDAR
}

\author{
NGUYEN XUAN TUAN, DINH VAN TRUNG, AND NGUYEN THANH BINH \\ Institute of Physics, Vietnam Academy of Science and Technology \\ BUI VAN HAI \\ Le Quy Don Technical University \\ E-mail: nxtuan@grad.iop.vast.ac.vn \\ Received 04 April 2014 \\ Accepted for publication 24 May 2014
}

\begin{abstract}
The nitrogen molecular density and temperature profiles of the upper troposphere are measured by a Raman lidar system in Hanoi over the range from $3 \mathrm{~km}$ to $19 \mathrm{~km}$. The spatial and temporal resolutions of profiles are $60 \mathrm{~m}$ and $1 \mathrm{~h}$, respectively. The nitrogen molecular density profiles are directly calculated from the range-corrected lidar signal. The temperature profiles are derived from the molecular density profile based on the assumptions of the hydrostatic equilibrium, the ideal-gas law and a fixed nitrogen molecule ratio in the atmosphere. The results of our lidar measurements show good agreement with the MSISE-90 atmospheric model. The maximum errors of density and temperature measurements are 6\% and 7\%, respectively. We estimated the height of tropopause in Hanoi about $16 \mathrm{~km}$ from the derived temperature profile. The measured density and temperature profiles from this Raman lidar can be used for studying the trends and characteristics of the upper troposphere in Hanoi.
\end{abstract}

Keywords: upper troposphere, temperature profile, Raman lidar.

\section{INTRODUCTION}

Temperature is the main parameter describing the dynamic state of the atmosphere, it relates to the form of cloud, controls the rate of chemical reaction, balances of the radiation. It appears in the forecast of weather and the climate and is the most important parameter in the weather prediction models. The atmospheric temperature depends on geographic location and time, so it is difficult to determine exactly in real time. The atmospheric temperature profile is often measured by the radiosonde. This is still the main technique to predict the in situ temperature of the atmosphere in the weather forecast. However, this technique is limited by the spatial and temporal resolutions, the measured height and interval. In addition, balloon and sensor in this technique are only used at one time.

Ever since, lidar (light detection and ranging) was applied in studying the atmosphere, it has become an advanced remote sensing technique. Lidar technique has also been used to measure the atmospheric temperature profile. The first atmospheric temperature measurement using the Rayleigh lidar technique were carried out by A. Hauchecorne and M. L. Chanin in 1980 [1]. The (C)2014 Vietnam Academy of Science and Technology 
atmospheric temperature profile is derived from the air molecular density profile based on the assumptions of the ideal gas and the hydrostatic equilibrium. They have obtained the atmospheric temperature profiles from $30 \mathrm{~km}$ to $70 \mathrm{~km}$. However, this lidar technique can only be applied if the contamination of aerosol in the atmosphere is not significant. This method has larger errors in case of working in the lower atmospheric layers because it could not separate exactly molecule and aerosol backscattering [2].

In order to measure the temperature in the lower atmospheric region, the lidar technique using the vibration-rotation Raman backscatter of nitrogen molecule is used. This technique also uses the above assumptions and a fixed nitrogen mixing ratio to obtain the temperature profile. Because the Raman backscattering signal of nitrogen molecule does not contain the backscattering of aerosol, the molecule density can be directly derived from Raman lidar signal by the rearrangement of the Raman lidar equation. The first measurements using this lidar technique were carried out by Philippe Keckhut et al. in 1990 and they have obtained the temperature profile from $12 \mathrm{~km}$ to $30 \mathrm{~km}$ [4]. They had corrected the transmission of molecule by using the CIRA-86 atmospheric model and the transmission of aerosol by using other lidar database.

In this paper we present the atmospheric temperature measurement using Raman lidar technique in Hanoi. We have used the proposed method by A. Ansmann et. al. [3] to correct the transmission of aerosol and the iterative method of W. N Chen et. al [2] to correct the transmission of molecule. The design, the specification and the data analysis of the Raman lidar system are also presented. The errors of density and temperature measurements are estimated and discussed in detail. The difference between the lidar results and the MSISE-90 atmospheric model in Hanoi is also compared and discussed.

\section{THE MEASUREMENT BY RAMAN LIDAR}

\section{II.1. Raman lidar system}

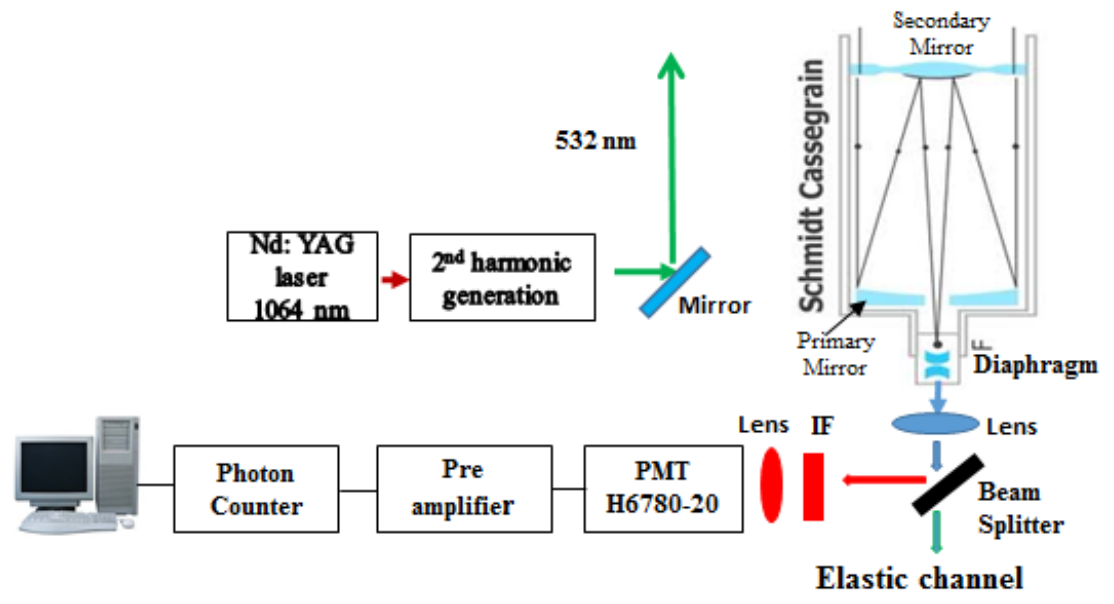

Fig. 1. Schematic block of Raman lidar system for temperature measurements. 
The Fig. 1 shows the schematic block of our Raman lidar system located in Hanoi, Vietnam $\left(21^{\circ} 2^{\prime} 0^{\prime \prime} \mathrm{N}, 105^{\circ} 51^{\prime} 00^{\prime \prime} \mathrm{E}\right.$, about $20 \mathrm{~m}$ above sea level). The Raman lidar system is set up based on the monostatic-biaxial configuration consisting of two parts: transmitter and receiver. The transmitter employs a second harmonic Nd:YAG pulsed laser at the wavelength of $532 \mathrm{~nm}$ with the maximum energy per pulse of $180 \mathrm{~mJ}$ and the repetition rate of $10 \mathrm{~Hz}$. The laser beam with the diameter of $6 \mathrm{~mm}$ is directed vertically to the sky by a steering mirror. Raman backscattering light (lidar signal) of the nitrogen molecule from the atmosphere is collected by a Schmidt-Cassegrain optical telescope in the diameter of $25 \mathrm{~cm}$. Raman lidar signal is filtered through the combination of a longpass interference filter FL-550 and a bandpass interference filter FL-610-10 nm from Thorlabs (USA). A photomultiplier tube H6780-20 (Hamamatsu, Japan) is employed to convert the light signal to an electrical signal. Photon single pulses are amplified by a preamplifier and counted by a fast photon counter, which is developed around a USB digital oscilloscope Picoscope 5204 (Picotech, UK). Histogram of counted photon number in each time bin is accummulated in real time by a program developed in Labview environment. Data files are stored in the format of *.txt for convenience in the analysis. The specification of our Raman lidar system is summarized in the Table 1.

Table 1. Specification of our Raman lidar system

\begin{tabular}{|l|l|}
\hline \multicolumn{1}{|c|}{ Transmitter } & \multicolumn{1}{c|}{ Receiver } \\
\hline - Laser Nd: YAG wavelength at $532 \mathrm{~nm}$ & - Telescope Schmidt - Cassegrain \\
- Energy per pulse (max): $180 \mathrm{~mJ}$ & - Diameter of telescope: $25 \mathrm{~cm}$ \\
- Pulse duration: $5 \mathrm{~ns}$ & - FOV: 1 mrad \\
- Repetition rate: $10 \mathrm{~Hz}$ & - PMT H6780 -20 Hasamatsu \\
- Beam divergence: $0.5 \mathrm{mrad}$ & - Photon Counter Module $8 \mathrm{bit}$ \\
\hline
\end{tabular}

\section{II.2. Signal measurement and analysis}

The Raman lidar system has to operate during night time to avoid the solar background light and measurements are carried out in the clear sky condition when the attenuation due to clouds is negligible. In our measurements, the photon count is acquired in 10 minute interval which corresponds to 6000 laser shots. The signal data files could be averaged together during off-line data analysis to increase signal-to-noise ratio (SNR). The spatial resolution (time bin) is set initially at $8 \mathrm{~ns}$ or $1.2 \mathrm{~m}$. The flow chart of data analysis algorithm for the inversion of Raman temperature lidar is showed in Fig. 2. The algorithm is divided into two stages. In the first stage, we investigate the 10 minute signal files to validate for temperature calculation. The raw lidar signal is subtracted by the background noise. The lidar signal is then multiplied by the square of the height to obtain the range-corrected lidar signal. Because the Raman range-corrected lidar signal profile is directly proportional to the nitrogen molecular number density profile, we could examine the reliability of measurement by comparison between the measured lidar signal profile and the nitrogen molecular profile from the MSISE-90 atmospheric model. The Raman lidar signal files, which are heavily contaminated by cloud, are rejected in the temperature calculation. An example about one validated signal file in 10 minutes is shown in Fig. 3. The left frame is the lidar signal and the right frame is the Raman range-corrected lidar signal in logarithmic scale compared with the molecular number density taken from the MSISE-90 atmospheric model [6]. 
The Raman lidar signal is then used to calculate the extinction of aerosol to compensate for the transmission of aerosol in the atmosphere.

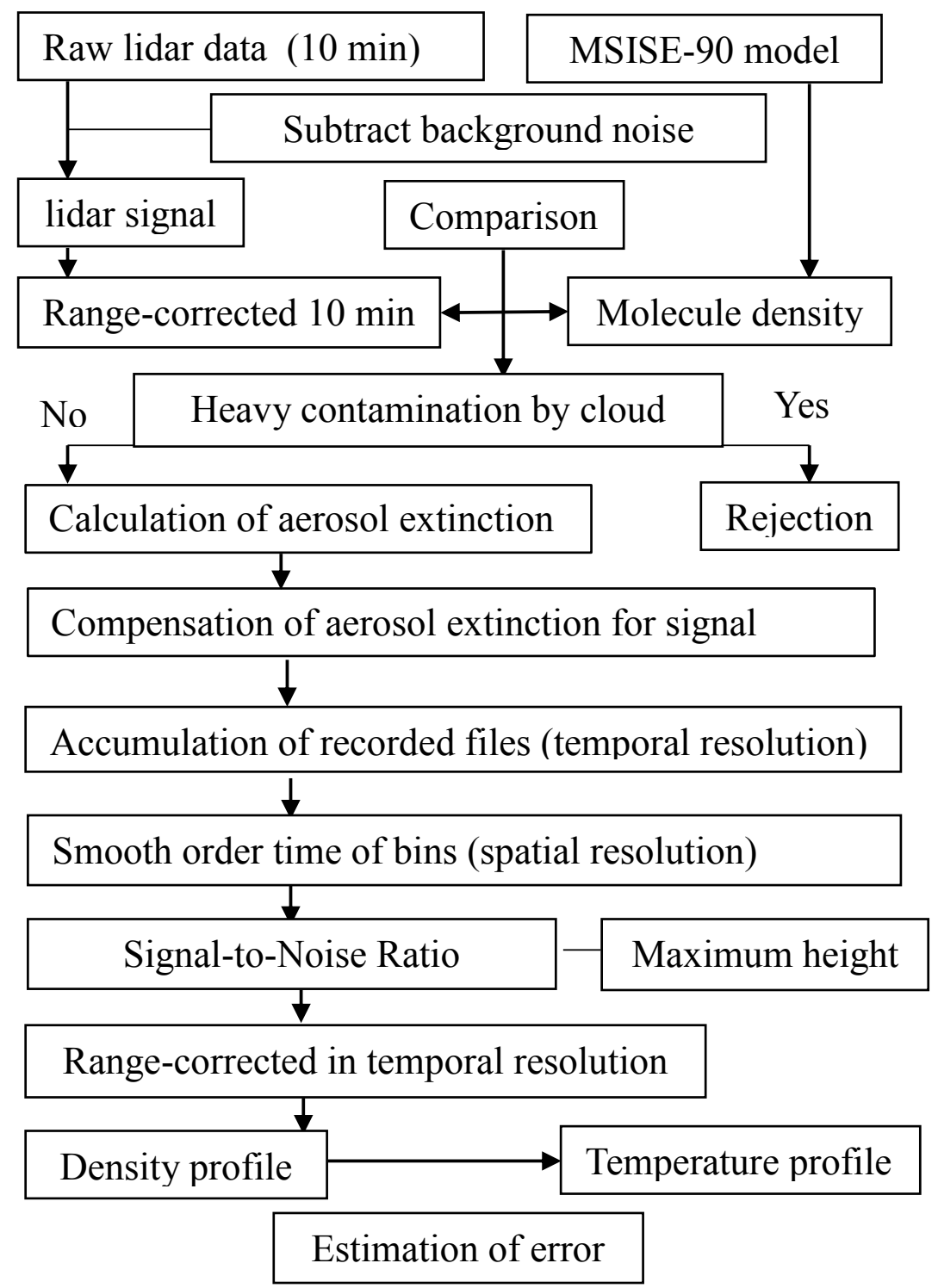

Fig. 2. The flow chart of algorithm for Raman temperature lidar.

In the second stage, several 10 minute validated signal files are accumulated and smoothed order time for increasing the signal-to-noise ratio (SNR). In our measurement, six data files, which are equal to the 1 hour temporal resolution, are accumulated for calculating the atmospheric temperature profile and smoothed order time 50 bins make the spatial resolution of $60 \mathrm{~m}$ for the profile. 
The maximum height of the profile is determined when the SNR is higher than 1. The SNR can be expressed by the equation as follows [7]:

$$
S N R=\frac{P_{\text {signal }}}{\sqrt{P_{\text {signal }+2 P_{b g}}}}
$$

where $P_{\text {signal }}$ is the photon number after subtracting the background noise, $P_{b g}$ is the background noise. The Raman lidar signal of nitrogen molecule is after correcting by the attenuation of aerosol that will be multiplied the range squared to get the range-corrected lidar signal in 1 hour. This range-corrected lidar signal is used to derive the molecular number density profile and the temperature profile. Finally, the errors of measurement are estimated. An example of the range-corrected Raman lidar signal and its signal-to-noise ratio in 1 hour are shown in Fig. 4.
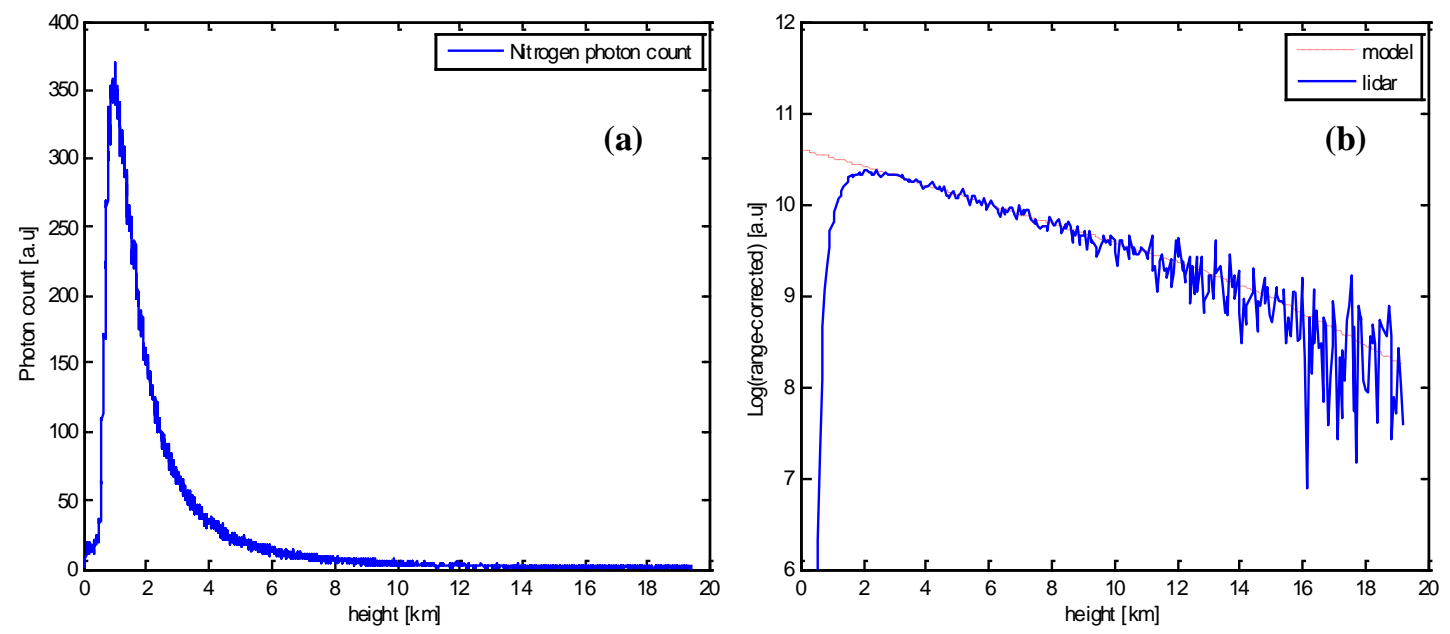

Fig. 3. Raw Raman lidar signal (a), and the range-corrected lidar signal accumulated in 10 minutes (b) in 26 June 2013 in Hanoi, the dotted line is the MSISE-90 molecular density.

\section{DETERMINATION OF TEMPERATURE USING RAMAN LIDAR}

\section{III.1. Derivation of temperature profile}

The Raman backscattering signal of nitrogen molecule from the atmosphere is described by the Raman lidar equation as follows [3]:

$$
P\left(\lambda_{R}, z\right)=P\left(\lambda_{0}\right) \frac{A_{T} \cdot O(z)}{z^{2}} \beta_{N}\left(\lambda_{R}, z\right) \cdot \exp \left(-\left[\alpha^{m o l}\left(\lambda_{0}, \lambda_{R}, z\right)+\alpha^{a e r}\left(\lambda_{0}, \lambda_{R}, z\right)\right]\right) .
$$

where $P\left(\lambda_{R}, z\right)$ is the photon count from Raman backscattering of nitrogen molecule, $P\left(\lambda_{0}\right)$ is the number of photon in the laser pulse $\lambda_{0}, \lambda_{R}$ are the laser wavelength and Raman backscattering wavelength, respectively. AT is the system calibration factor (includes the area of telescope and the efficiencies of detector). $O(z)$ is the overlap function between the laser beam and the axis of 

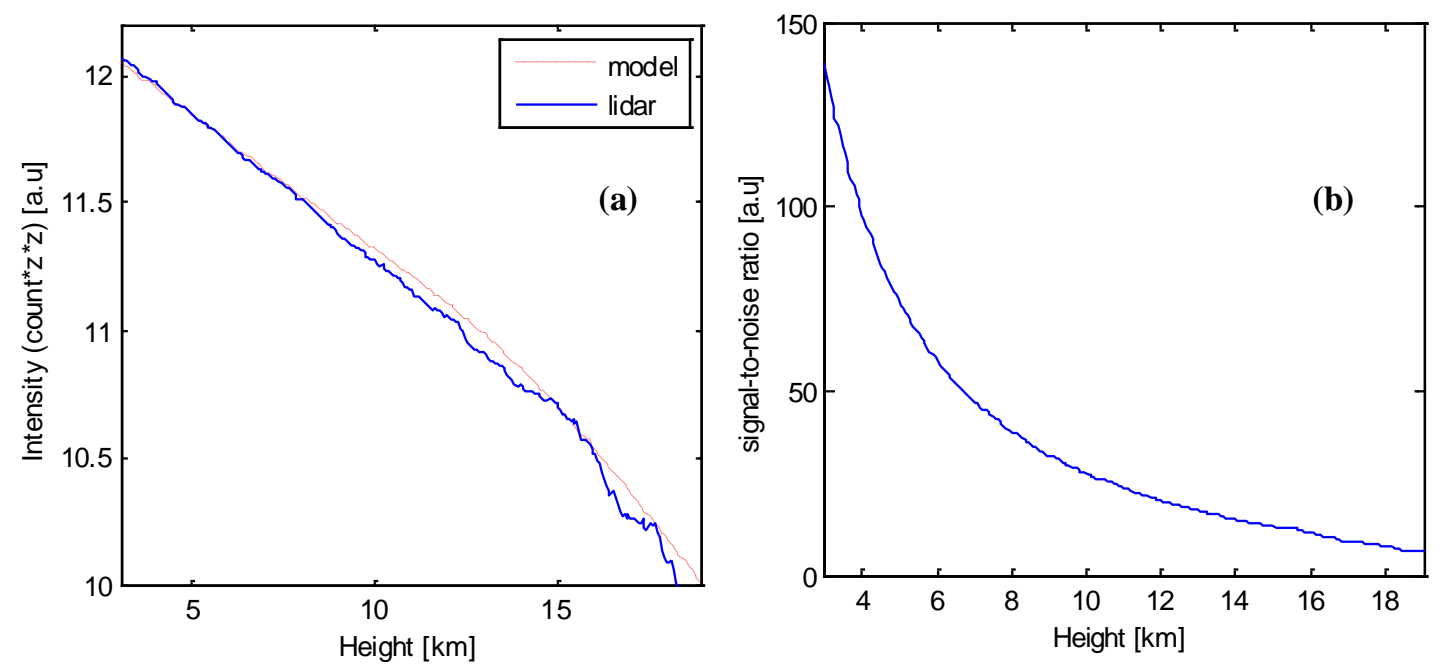

Fig. 4. The range-corrected lidar signal (a) and the Raman signal-to-noise ratio (b) profiles accumulated in $1 \mathrm{~h}$ in 26 June 2013 in Hanoi.

the telescope. $\beta_{N}\left(\lambda_{R}, z\right)$ is the Raman backscattering coefficient of nitrogen molecule described by the equation [3]:

$$
\beta_{N}\left(\lambda_{R}, z\right)=\rho_{N}(z) \frac{d \sigma_{N}(\pi)}{d \Omega} .
$$

where $\rho_{N}(z)$ is the number density of nitrogen molecule, $\frac{d \sigma_{N}(\pi)}{d \Omega}$ is the range-independence differential Raman backscattering cross-section of nitrogen. $\alpha^{\text {mol }}\left(\lambda_{0}, \lambda_{R}, z\right), \alpha^{a e r}\left(\lambda_{0}, \lambda_{R}, z\right)$ are the atmospheric round-trip extinction coefficient of molecule and aerosol, respectively, expressed by the equations [3]:

$$
\begin{aligned}
& \alpha^{\mathrm{mol}}\left(\lambda_{0}, \lambda_{R}, z\right)=\quad \alpha^{\mathrm{mol}}\left(\lambda_{0}, z\right)+\alpha^{\mathrm{mol}}\left(\lambda_{R}, z\right)=\left[\sigma^{\mathrm{mol}}\left(\lambda_{0}\right)+\sigma^{\mathrm{mol}}\left(\lambda_{R}\right)\right] \int_{0}^{z} \rho^{\mathrm{mol}}\left(z^{\prime}\right) d z^{\prime}, \\
& \alpha^{\text {aer }}\left(\lambda_{0}, \lambda_{R}, z\right)=\alpha^{\text {aer }}\left(\lambda_{0}, z\right)+\alpha^{\text {aer }}\left(\lambda_{R}, z\right)=\left[\sigma^{\text {aer }}\left(\lambda_{0}, z\right)+\sigma^{\text {aer }}\left(\lambda_{R}, z\right)\right] \int_{0}^{z} \rho_{a e r}\left(z^{\prime}\right) d z^{\prime},
\end{aligned}
$$

where $\alpha^{m o l}\left(\lambda_{0}, z\right), \alpha^{m o l}\left(\lambda_{R}, z\right)$ are the one-trip extinction coefficient of molecule at the laser wavelength and the Raman wavelength, respectively. $\alpha^{a e r}\left(\lambda_{0}, z\right), \alpha^{a e r}\left(\lambda_{R}, z\right)$ are the one-trip extinction coefficient of aerosol at the laser wavelength and the Raman wavelength. $\sigma^{\mathrm{mol}}\left(\lambda_{0}\right), \sigma^{\mathrm{mol}}\left(\lambda_{R}\right)$ are the range-independence backscattering cross-section of molecule at the laser wavelength and the Raman wavelength. $\sigma^{a e r}\left(\lambda_{0}, z\right), \sigma^{a e r}\left(\lambda_{R}, z\right)$ are the range-dependence backscattering crosssection of aerosol at the laser wavelength and the Raman wavelength. $\rho_{\text {mol }}\left(z^{\prime}\right), \rho_{\text {aer }}\left(z^{\prime}\right)$ are the number density of molecule and aerosol, respectively. $\sigma^{a e r}\left(\lambda_{0}, z\right), \sigma^{a e r}\left(\lambda_{R}, z\right)$ are related together the equation [3]:

$$
\frac{\left(\sigma^{a e r}\left(\lambda_{R}, z\right)\right)}{\left(\sigma^{a e r}\left(\lambda_{0}, z\right)\right)}=\left(\lambda_{0} / \lambda_{R}\right)^{\gamma}
$$


$\gamma$ is the wavelength dependence of aerosol backscattering. It is often chosen to be 1 in the upper troposphere because the size of aerosol is small. The one-trip extinction coefficient of aerosol could be determined following the proposed method by A. Ansmann et al. (1992) described by the equation [3]:

$$
\alpha^{\text {aer }}\left(\lambda_{0}, z\right)=\frac{\frac{d}{d z}\left\{\ln \left[\frac{\rho_{N}^{r e f}(z)}{P\left(\lambda_{R}, z\right) \cdot z^{2}}\right]\right\}-\left(\alpha^{m o l}\left(\lambda_{0}, z\right)+\alpha^{m o l}\left(\lambda_{R}, z\right)\right)}{1+\left(\frac{\lambda_{0}}{\lambda_{R}}\right)^{\gamma}}
$$

where $\alpha^{\text {mol }}\left(\lambda_{0}, z\right), \alpha^{m o l}\left(\lambda_{R}, z\right)$ are determined from Eqs. (4) and (5) by using the molecular number density of other data (radiosonde or model).

The efficient Raman lidar signal factor $P_{\text {eff }}\left(\lambda_{\mathrm{R}}, z\right)$ is defined by the Raman lidar signal after corrected by the aerosol extinction in the atmosphere

$$
P_{\text {eff }}\left(\lambda_{R}, z\right)=P\left(\lambda_{R}, z\right) \cdot\left[\exp \left(-\left[\alpha^{\text {aer }}\left(\lambda_{0}, \lambda_{R}, z\right)\right]\right)\right]^{-1} .
$$

The absolute number density of nitrogen molecule is determined by rearranging the Raman lidar equation (Eq. (2)). It is described as follows:

$$
\rho_{N}(z)=C \times P_{e} f f\left(\lambda_{R}, z\right) \cdot z^{2} \times\left[\exp \left(-\alpha^{m o l}\left(\lambda_{0}, \lambda_{R}, z\right)\right)\right]^{-1}
$$

where $C$ is the normalization factor. It depends on parameters of lidar system (parameters of laser beam, telescope area, efficiency of the lidar system) and the parameters of atmospheric (the type of detected signal, the height resolution of measured data, the aerosol present level of the atmosphere). $C$ is quantified by normalizing $\rho_{N}(z)$ to the pre-specified density $\rho_{N}^{\bmod }\left(z_{n o r m}\right)$ at the normalization height $z_{n o r m}$ that must be chosen higher than aerosol regions. After the normalization, we have [4]

$$
C=\rho_{N}^{\text {mod }}\left(z_{\text {norm }}\right) \times\left[P_{\text {eff }}\left(\lambda_{R}, z_{\text {norm }}\right) \times z_{\text {norm }}^{2}\right]^{-1} \times\left[\exp \left(-\alpha^{\text {mol }}\left(\lambda_{0}, \lambda_{R}, z_{\text {norm }}\right)\right)\right] .
$$

The one-trip extinction coefficient of molecule in the Eq. (9) is unknown but it can be calculated by the iterative method based on Eqs. (4) and (9). We start with a molecular number density from the MSISE-90 model to obtain the one-trip extinction coefficient of aerosol following the Eq. (7) and nitrogen molecule density following the Eq. (9). In the second step, the new molecule density profile is used to calculate the one-trip extinction coefficient of molecule following the Eq. (9). In the third step, the first step and the second step are iterated and the molecule number density will converge after some iterative steps.

The relative fixed number density of nitrogen molecules in the upper troposphere is compared to the total atmospheric molecule (about 78\%). Based on the assumptions of the hydrostatic equilibrium and the ideal-gas law, the atmospheric temperature can be derived from the nitrogen molecular number density as follows [2]:

$$
T(z)=\frac{\rho_{N}\left(z_{\text {ref }}\right)}{\rho_{N}(z)} \times T\left(z_{\text {ref }}\right)+\frac{M}{R \times \rho_{N}(z)} \int_{z}^{z_{\text {ref }}} \rho_{N}\left(z^{\prime}\right) \times g\left(z^{\prime}\right) d z^{\prime} .
$$

where $g(z)$ is the gravitational acceleration, $M$ is the mean atmospheric molecular weight, $R$ is the molar gas constant. $T\left(z_{\text {ref }}\right)$ is the tie-on reference temperature obtained from other measurement data at the reference height $z_{r e f}$. The reference height is often chosen where the lidar signal could be reliable with high enough signal-to-noise ratio. 


\section{III.2. Estimation of errors of temperature}

Errors of temperature measurement of Raman lidar might originate from sources such as: uncertainty of aerosol, absorption of ozone, statistical noise, the selected tie-on temperature, the contamination of cloud.

The uncertainty of the absorption of ozone in the upper troposphere is negligible because the ozone concentration in the troposphere is small [2]. The error is caused by selection of the tie-on temperature $T\left(z_{\text {ref }}\right)$ can be derived from Eq. (10):

$$
\Delta T(z) \approx \frac{\rho\left(z_{r e f}\right)}{\rho(z)} \Delta T\left(z_{r e f}\right)
$$

where $\Delta T\left(Z_{r e f}\right)$ is the uncertainty of the tie-on reference temperature. Our lidar system operates in the photon counting mode, so the photon noise obeys the Poisson statistics. The error is caused by statistical noise can be expressed by equation [2]

$$
\Delta T(z) \approx \frac{T(z)}{\sqrt{m \cdot P\left(\lambda_{R}, z\right)}},
$$

where $m$ is number of laser pulses, $P\left(\lambda_{R}, z\right)$ is the backscattering photon counts of nitrogen. We can reduce the statistical noise error by sacrificing the spatial and temporal resolutions. The temperature error due to the uncertainty from the aerosol extinction is given by the equation:

$$
\Delta T \leq T\left\{\left[\frac{\Delta \exp \left(-\alpha^{a e r}\left(\lambda_{0}, \lambda_{R}, z\right)\right)}{\exp \left(-\alpha^{a e r}\left(\lambda_{0}, \lambda_{R}, z\right)\right)}\right]^{2}\right\}
$$

Our previous investigation by lidar measurement showed that the cirrus clouds in Hanoi are often located at about $16 \mathrm{~km}$ [8]. The top cirrus cloud could be predicted by comparison the Raman range-corrected profile with the nitrogen molecular profile of MSISE-90 atmospheric model in logarithmic scale.

\section{RESULTS AND DISCUSSIONS}

The Raman range corrected lidar signal profile was accumulated for $1 \mathrm{~h}$ from $7 \mathrm{~h} 50 \mathrm{PM}$ to $8 \mathrm{~h} 50 \mathrm{PM}$ in 26 June 2013 in Hanoi and we use this data to derive the molecular nitrogen number density profile. In order to decrease distortion due to the rapid decay of the lidar signal profile, the last $5 \mathrm{~km}$ (from the $14 \mathrm{~km}$ to the $19 \mathrm{~km}$ ) signal is smoothed in the logarithmic scale following the weighted linear least square method. Result of nitrogen molecular density profile from lidar measurement is presented in figure 5. The lidar profile starts from $3 \mathrm{~km}$ and finishes at $19 \mathrm{~km}$ with the spatial resolution is $60 \mathrm{~m}$. The top height is chosen where the signal-to-noise ratio is higher than 6 . The errors of density consist of the photon statistic noise and the uncertainty of aerosol. The maximum error of density is determined smaller than $6 \%$. The nitrogen density profile from our lidar measurement is compared with the nitrogen molecular density profile of the MSISE-90 atmospheric model. The difference between lidar and model results could be investigated together by the molecule deviation ratio as follows:

$$
R_{\rho}(z)=\frac{\left|\rho_{\text {model }}(z)-\rho_{\text {lidar }}(z)\right|}{\rho_{\text {model }}(z)} * 100 \%,
$$



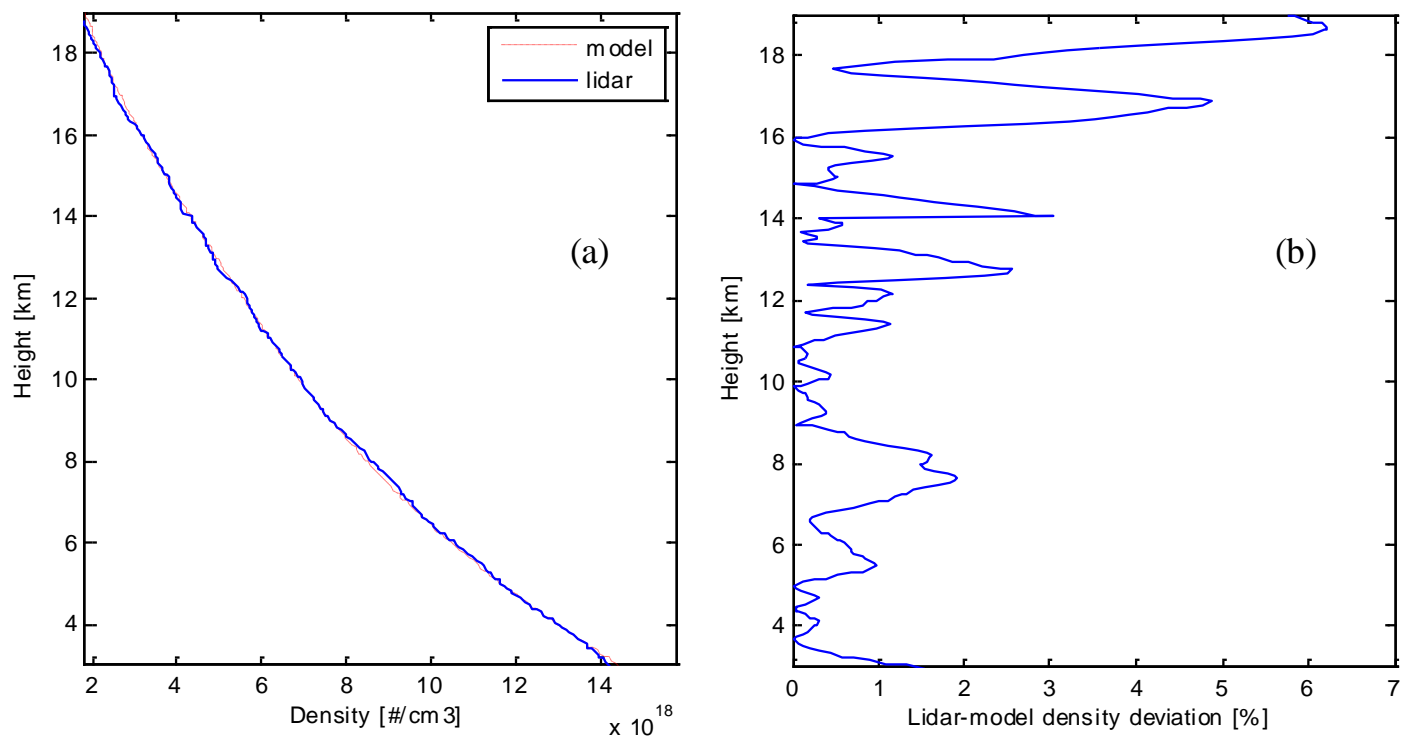

Fig. 5. The nitrogen molecular density profile derived from Raman lidar measurement compared to MSISE-90 atmospheric model (a) and the lidar-model molecular deviation ratio profile (b) in night 26 June 2013 in Hanoi.
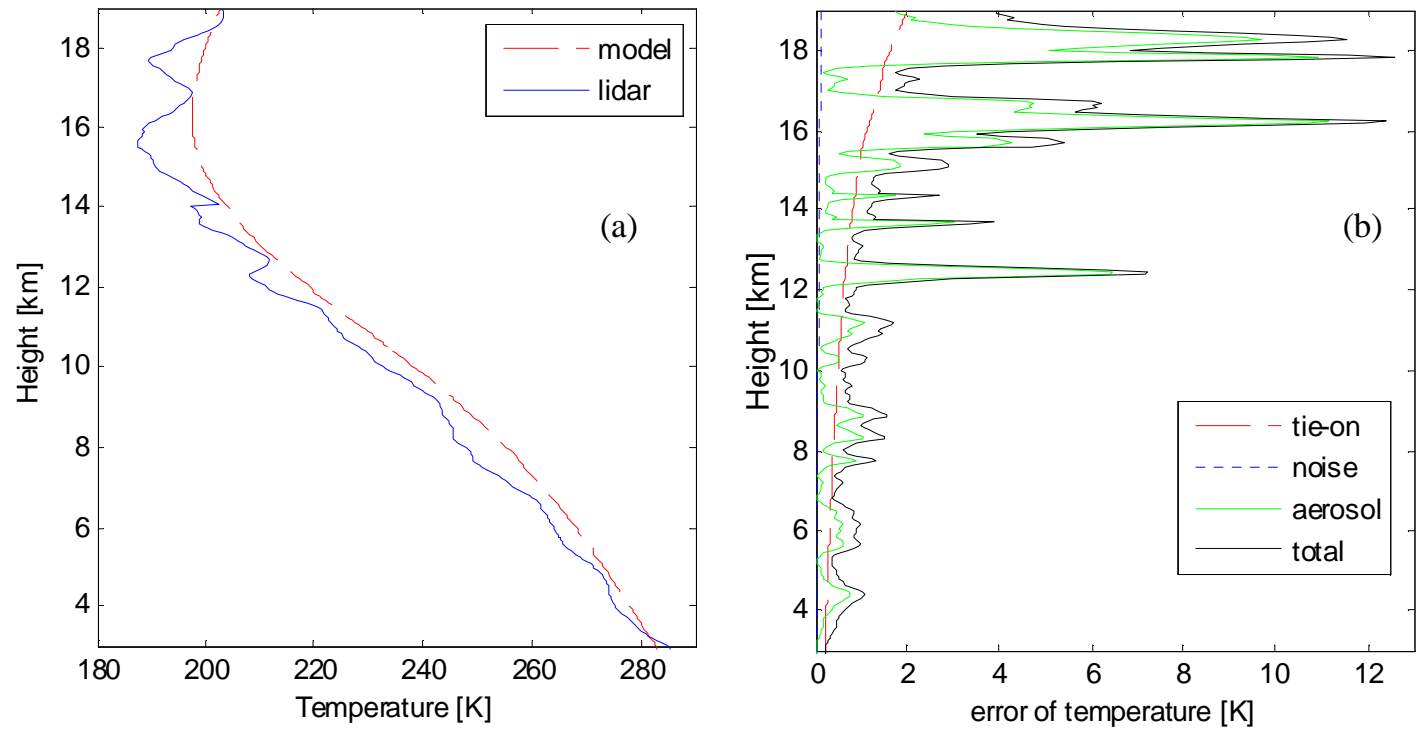

Fig. 6. The temperature profile (a) and the temperature error profile (b) obtained from inversion of Raman lidar signal in night 26 June 2013 in Hanoi.

where $\rho_{\text {model }}(z)$ is the number density of model, $\rho_{\text {lidar }}(z)$ is the number density of lidar. The maximum deviation ratio is smaller than $7 \%$. The good agreement between two profiles indicates the reliability in our measurement. 

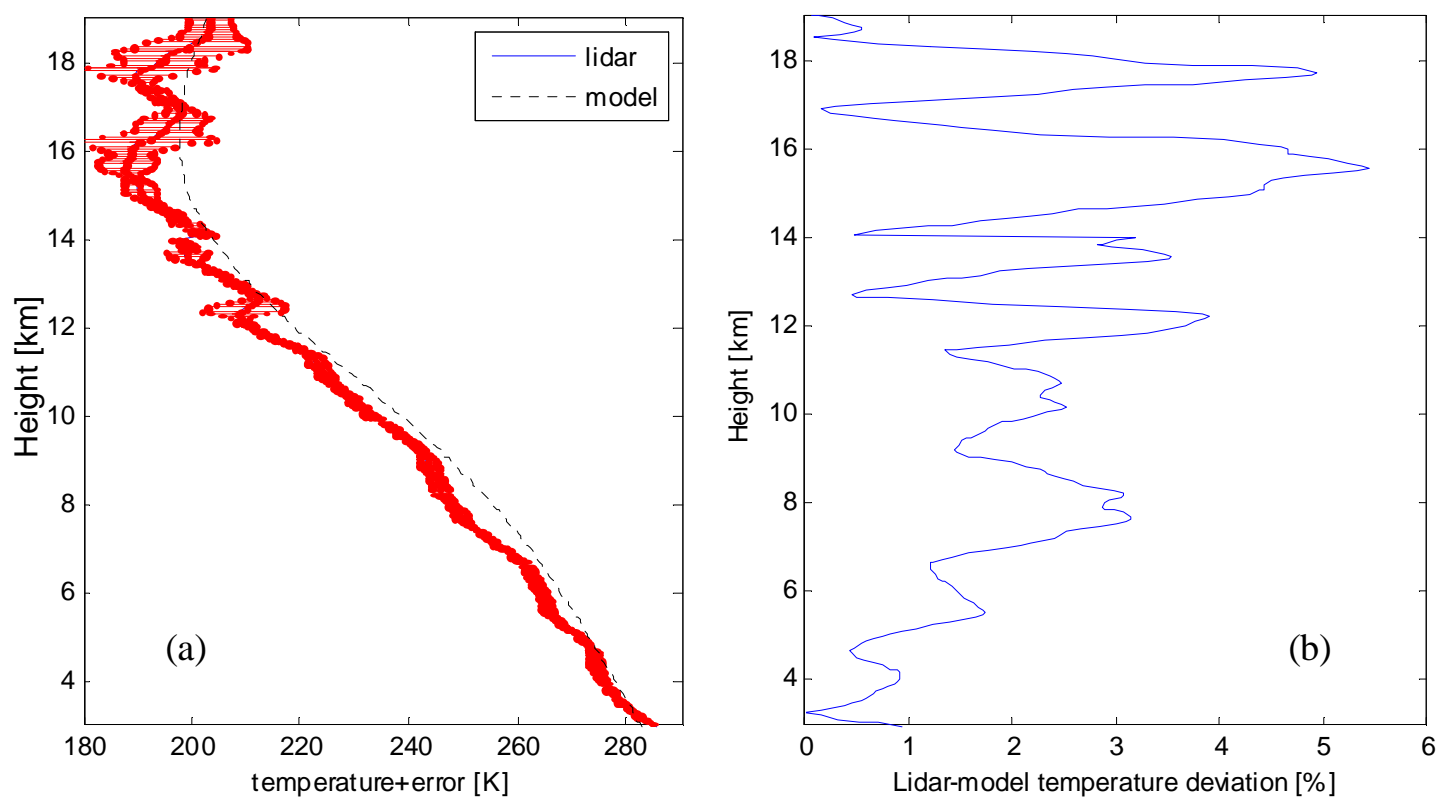

Fig. 7. The temperature and error bar profile (a) and the lidar-model temperature deviation ratio profile (b) obtained from Raman lidar measurement in night 26 June 2013 in Hanoi.

The atmospheric temperature profile is calculated from the above nitrogen density profile by the method described in Sec. III. The results of the upper tropospheric temperature profile and temperature errors profile in Hanoi from our Raman lidar measurement are shown in Fig. 6(a). The height, resolution of the temperature are the same as the density profile. The tie-on reference temperature from the MSISE-90 atmospheric model is chosen at the maximum height of the lidar signal profile. The temperature profile from our lidar measurement is also compared with the temperature profile of the MSISE-90 atmospheric model in order to validate the measured results. The agreement between the calculated and model profiles is found to be satisfactory.

The errors of temperature in our lidar measurement originate from the tie-on reference temperature, the photon noise, and the uncertainty of aerosol shown in the Fig. 6 (b). The result indicates that the error due to the uncertainty of aerosol is the largest. This error varies strongly from $14 \mathrm{~km}$ to $19 \mathrm{~km}$. The maximum temperature error determined from the profile is smaller than $7 \%$.

The temperature profile from lidar measurement is compared with the temperature profile from MSISE-90 atmosphere model shown in Fig. 7. The temperature deviation between lidar and model is investigated through the lidar-model temperature deviation ratio by the equation:

$$
R_{T}(z)=\frac{\left|T \rho_{\text {model }}(z)-T_{\text {lidar }}(z)\right|}{T_{\text {model }}(z)} * 100 \% .
$$

where $T_{\text {model }}(z)$ is the temperature of model, $T_{\text {lidar }}(z)$ is the temperature of lidar. The maximum deviation is smaller than $6 \%$. 
Our Raman temperature lidar measurement indicates the existence of the tropopause in atmosphere in Hanoi, its position is located at a height of $16 \mathrm{~km}$ to $18 \mathrm{~km}$. The tropopause, the located atmospheric area divided the troposphere and stratosphere, relates to the abundance of ozone and the exchange and coupling processes between the troposphere and the stratosphere. So, it gets the special interest in atmospheric research. Study about the temperature trend and structure of the tropopause in Hanoi by our lidar measurement will be reported in future publications.

In a previously published paper [8], we had investigated the top cirrus cloud in Hanoi by the combined Raman-elastic lidar and the relationship between the top cirrus cloud and the tropopause. The temperature profile is employed from radiosonde measurement on the same day to indicate the tropopause. However, the time of lidar and radiosonde measurements is different. We will report study of the characteristics of the top cirrus cloud and the tropopause in Hanoi by simultaneous lidar measurement in future publications.

\section{CONCLUSIONS}

We have presented measurements of the density and temperature profiles in the upper troposphere in Hanoi using the Raman lidar. The density of the molecular nitrogen profile is calculated from the range-corrected lidar signal accumulated in 1 hour. Then, the temperature profile is derived from the density profile by using the ideal gas and the hydrostatic equilibrium. The profiles had the bottom at $3 \mathrm{~km}$ and the top at $19 \mathrm{~km}$ with the resolution of $60 \mathrm{~m}$. The density and temperature profiles from lidar measurements show good agreement with the profiles of the MSISE-90 atmospheric model. The maximum error of density and temperature are determined to be smaller than $6 \%$ and $7 \%$, respectively. The main error in this lidar technique is the uncertainty of the aerosol extinction. The temperature profile might indicate the existence of the tropopause in Hanoi at the neighbor height from $16 \mathrm{~km}$ to $18 \mathrm{~km}$. The measured density and temperature profiles from this Raman lidar technique can be used for studying the trends and characteristics of the upper troposphere in Hanoi.

\section{ACKNOWLEDGEMENTS}

We acknowledge the support from Institute of Physics, VAST for the development of lidar system.

\section{REFERENCES}

[1] A. Hauchecorne and M. L. Chanin, Geophys. Res. Lett.7 (1980) 565-568.

[2] W. N. Chen, C. C. Tsao, J. B. Nee, Journal of Atmospheric and Solar-Terrestrial Physics 66 (2004) 39-49.

[3] A. Ansmann, M. Riebesell, U. Wandinger, C. Weitkamp, W. Lahmann, and W. Michaelis, Applied Physics B 55 (1992) $18-28$.

[4] Philippe Keckhut, M. L. Chanin, and A. Hauchecorne, Applied Optics 29(34) (1990) 5182-5186.

[5] Keith D. Evans, S. Harvey Melfi, Richard A. Ferrare, and David N. Whiteman, Applied Optics 36(12) (1997) 2594-2602.

[6] http://omniweb.gsfc.nasa.gov/vitmo/msis_vitmo.html

[7] B. Heese, H. Flentje, D. Althausen, A. Ansman, and S. Frey, Atmospheric Measurement Techniques 3(4) (2010) 3907-3924.

[8] Bui Van Hai, Dinh Van Trung, Nguyen Xuan Tuan, Dao Duy Thang, and Nguyen Thanh Binh, Communications in Physics 22(4) (2012) 357-364. 